\title{
An efficient wave extrapolation method for anisotropic media with tilt
}

\author{
Umair bin Waheed* and Tariq Alkhalifah* \\ Physical Sciences and Engineering Division, King Abdullah University of Science and Technology, Thuwal 23955-6900, Saudi Arabia
}

Received May 2014, revision accepted October 2014

\begin{abstract}
Wavefield extrapolation operators for elliptically anisotropic media offer significant cost reduction compared with that for the transversely isotropic case, particularly when the axis of symmetry exhibits tilt (from the vertical). However, elliptical anisotropy does not provide accurate wavefield representation or imaging for transversely isotropic media. Therefore, we propose effective elliptically anisotropic models that correctly capture the kinematic behaviour of wavefields for transversely isotropic media. Specifically, we compute source-dependent effective velocities for the elliptic medium using kinematic high-frequency representation of the transversely isotropic wavefield. The effective model allows us to use cheaper elliptic wave extrapolation operators. Despite the fact that the effective models are obtained by matching kinematics using high-frequency asymptotic, the resulting wavefield contains most of the critical wavefield components, including frequency dependency and caustics, if present, with reasonable accuracy. The methodology developed here offers a much better cost versus accuracy trade-off for wavefield computations in transversely isotropic media, particularly for media of low to moderate complexity. In addition, the wavefield solution is free from shear-wave artefacts as opposed to the conventional finite-difference-based transversely isotropic wave extrapolation scheme. We demonstrate these assertions through numerical tests on synthetic tilted transversely isotropic models.
\end{abstract}

Key words: Anisotropy, Modelling, Wave.

\section{INTRODUCTION}

Forward modelling lies at the heart of all inversion algorithms. The computational efficiency of these inversion schemes is usually bottlenecked by the modelling tool they resort to. Therefore, the development of highly efficient modelling algorithms can greatly impact modern-day computationally intensive inversion procedures.

The task of wavefield extrapolation refers to the advancement of wavefield in small steps through space or time using extrapolation operators that satisfy the wave equation. It is a key ingredient in seismic modelling, imaging, and fullwaveform inversion algorithms. For example, reverse time migration is able to image complex subsurface structures by

*E-mails: umairbin.waheed@kaust.edu.sa,tariq.alkhalifah@kaust.edu.sa directly solving the acoustic wave equation (Baysal, Kosloff, and Sherwood 1983; McMechan 1983; Fletcher, Du, and Fowler 2009). It relies on accurate and efficient forward and backward extrapolation of waves in time.

Despite all the complexities involved in extrapolating wavefields in anisotropic media, namely, the shear-wave artefact and stability constraints, the improved images obtained by incorporating anisotropy (Zhou et al. 2004; Huang et al. 2008) justify the efforts. Wavefields in anisotropic media are well described by the anisotropic elastic wave equation (Crampin 1984). However, in practice, we usually do not deal with information related to shear waves and prefer to use scalar wavefields, particularly for conventional imaging applications. Therefore, the acoustic anisotropic wave equation is now heavily employed in industry for subsurface imaging algorithms. 
A transversely isotropic (TI) model with tilted axis of symmetry (TTI) is regarded as one of the most effective approximations of the Earth's subsurface, especially for imaging purposes. The $\mathrm{P}$-wave phase velocity in three dimensions for TI media, under the acoustic assumption, depends on the symmetry axis velocity $v_{0}$, the normal moveout velocity $v_{\text {nmo }}$ (related to $v_{0}$ through Thomsen's $\delta$ parameter), and the anellipticity anisotropy parameter $\eta$. Moreover, it depends on the dip angle $\theta$ that the symmetry axis makes with the vertical and the azimuthal angle $\phi$ of the plane containing symmetry axis with respect to the $x$-axis (Tsvankin 1997).

The computational cost of a wavefield extrapolation algorithm is directly affected by the level of complexity in description of the medium properties. Including either anisotropy, attenuation, or poroelasticity or all of them in medium characterization can lead to significant increase in cost of solving the corresponding wave equation. However, embedding the kinematic and dynamic effects of these physical phenomena into a simpler model, requiring smaller number of parameters for characterization, can considerably reduce computational burden. The idea relies on obtaining simpler effective models that exhibit wave propagation behaviour similar to those present in the original model. Ideally, the wavefield obtained for the effective model would match both the phase and amplitude properties of the original model.

Alkhalifah et al. (2013) proposed the idea of using effective isotropic models for wave propagation in TI media. They obtained a source-dependent effective isotropic model by embedding kinematic effects of the anisotropic medium into the isotropic one using solution to the anisotropic eikonal equation. The effective model was then used to compute wavefields by using the much cheaper isotropic wavefield extrapolation operator. The cost of this additional step of solving the anisotropic eikonal equation is several times lower than computing the anisotropic wavefield solution (Alkhalifah et al. 2013). Therefore, the approach showed great promise in reducing computational cost of wavefield extrapolation in anisotropic media. Even though the resulting wavefield yielded a perfect kinematic match for the first arriving wave, kinematic content of later arrivals and amplitude information suffered from significant inaccuracies (Ibanez-Jacome, Alkhalifah, and Waheed 2014).

The cost of solving the wave equation for elliptically anisotropic media is very similar to that for the isotropic case. Therefore, in this paper, we propose effective elliptically anisotropic models for wave extrapolation in TI media. Compared with the effective isotropic model, the increase in number of parameters in representing the effective medium yields a better match of phase and amplitude contents at almost the same computational cost.

Moreover, the acoustic TI approximation of setting the shear-wave velocity along the symmetry axis to zero does not prevent existence of the unwanted shear-wave noise into the P-wave solution (Xu and Zhou 2014). This can lead to numerical instability of the wave extrapolation algorithm, in addition to causing uncertainty in interpretation. Therefore, it becomes vital to remove the shear-wave noise from the wavefield solution. Although it is possible to suppress the unwanted shear-wave artefacts through postprocessing filters (Zhang et al. 2009; Guan et al. 2011), these approaches lead to a significant increase in computational cost.

However, an elliptically anisotropic wave extrapolation algorithm does not suffer from such artefacts. The proposed formulation is completely free from shear-wave noise and therefore does not require costly noise filtering operations. In addition, the method can also be used for TI models with negative anellipticity values for which conventional extrapolation schemes have been found to be unstable.

The rest of the paper is organized as follows. We begin by describing theoretical foundations behind the idea of obtaining source-dependent effective elliptic models. This is followed by a description of the algorithm to compute approximate wavefields in TI media. Then, we will demonstrate applicability and usefulness of our proposed method on synthetic TTI models.

\section{THEOR Y}

\section{The TTI wave equation}

Let $P(x, z, t)$ be the seismic wavefield at a location $(x, z)$ at time $t$. Then, the acoustic wave equation for 2D TI media with vertical symmetry axis (VTI) is given as (Alkhalifah 2000):

$\frac{\partial^{4} P}{\partial t^{4}}=v_{n m o}^{2}(1+2 \eta) \frac{\partial^{4} P}{\partial t^{2} \partial x^{2}}+v_{0}^{2} \frac{\partial^{4} P}{\partial t^{2} \partial z^{2}}-2 \eta v_{n m o}^{2} v_{0}^{2} \frac{\partial^{4} P}{\partial x^{2} \partial z^{2}}$,

where $v_{0}$ is the $\mathrm{P}$-wave phase velocity, $v_{n m o}$ is the normal moveout (NMO) velocity, and $\eta$ is the anellipticity anisotropy parameter, all computed along the symmetry direction.

Tectonic movements of the crust and migration of salt bodies may rotate rocks and tilt the natural vertical orientation of the symmetry axis, resulting in a TTI model. 
By computing spatial wavenumbers in the rotated coordinate frame, we derive the wave equation for TTI medium to be:

$$
\begin{aligned}
\frac{\partial^{4} P}{\partial t^{4}}= & v_{n m o}^{2}(1+2 \eta) \\
& \times\left(\cos ^{2} \theta \frac{\partial^{4} P}{\partial t^{2} \partial x^{2}}+\sin ^{2} \theta \frac{\partial^{4} P}{\partial t^{2} \partial z^{2}}+\sin 2 \theta \frac{\partial^{4} P}{\partial t^{2} \partial x \partial z}\right) \\
& +v_{0}^{2}\left(\cos ^{2} \theta \frac{\partial^{4} P}{\partial t^{2} \partial z^{2}}+\sin ^{2} \theta \frac{\partial^{4} P}{\partial t^{2} \partial x^{2}}-\sin 2 \theta \frac{\partial^{4} P}{\partial t^{2} \partial x \partial z}\right) \\
& -2 \eta v_{n m o}^{2} v_{0}^{2}\left(\cos ^{2} \theta \sin ^{2} \theta\left(\frac{\partial^{4} P}{\partial x^{4}}+\frac{\partial^{4} P}{\partial z^{4}}\right)\right. \\
& +\left(2 \cos \theta \sin ^{3} \theta-2 \cos ^{3} \theta \sin \theta\right)\left(\frac{\partial^{4} P}{\partial x^{3} \partial z}+\frac{\partial^{4} P}{\partial x \partial z^{3}}\right) \\
& \left.+\left(\cos ^{4} \theta+\sin ^{4} \theta-4 \cos ^{2} \theta \sin ^{2} \theta\right) \frac{\partial^{4} P}{\partial x^{2} \partial z^{2}}\right)
\end{aligned}
$$

where $\theta$ is the layering dip angle measured with respect to the vertical.

\section{Effective vertical elliptically anisotropic model}

The wave equation for an elliptically anisotropic medium with vertical axis of symmetry (VEA) is obtained by setting $\eta=0$ in the VTI wave equation (1):

$\frac{\partial^{2} P}{\partial t^{2}}=v_{n m o}^{2} \frac{\partial^{2} P}{\partial x^{2}}+v_{0}^{2} \frac{\partial^{2} P}{\partial z^{2}}$.

It is noticeable that the extrapolation operator for VEA medium is much simpler and has been estimated to be at least three times computationally efficient than the one for 2D TTI media (Waheed and Alkhalifah 2014). However, elliptical anisotropy does not provide accurate wavefield or imaging for media of TI anisotropy. Therefore, embedding kinematic and dynamic effects due to $\eta$ and $\theta$ into $v_{0}$ and $v_{\text {nmo }}$ allows us to use the much simpler VEA wave extrapolation operator to obtain solution for the TTI wave equation. Here we focus on obtaining effective VEA models that match the kinematic behaviour of wave propagation in TTI media. An extension of this method has been proposed to match the amplitude information as well. For details, refer to Wang, Waheed, and Alkhalifah (2014). The kinematic matching is obtained through an additional step of solving the TTI eikonal equation. The cost of this additional step is negligible compared to solving for the TTI wavefield (Alkhalifah et al. 2013).

In order to derive effective velocities for VEA medium that match kinematic wave behaviour of TTI model, we con- sider the TTI eikonal equation (Stovas and Alkhalifah 2012):

$$
\begin{aligned}
& v_{n \text { по }}^{2}(1+2 \eta)\left(\cos \theta \frac{\partial \tau}{\partial x}+\sin \theta \frac{\partial \tau}{\partial z}\right)^{2} \\
& +v_{0}^{2}\left(\cos \theta \frac{\partial \tau}{\partial z}-\sin \theta \frac{\partial \tau}{\partial x}\right)^{2} \\
& \quad \times\left(1-2 \eta v_{n m o}^{2}\left(\cos \theta \frac{\partial \tau}{\partial x}+\sin \theta \frac{\partial \tau}{\partial z}\right)^{2}\right)=1,
\end{aligned}
$$

where $\tau(x, z)$ is the travel time measured from the source to a point with coordinates $(x, z)$.

Rewriting the TTI eikonal equation (4) after some manipulations yields:

$$
\begin{aligned}
& \frac{\left(v_{n m o}^{2}(1+2 \eta) \cos ^{2} \theta+v_{0}^{2} \sin ^{2} \theta\right)}{c(\tau)}\left(\frac{\partial \tau}{\partial x}\right)^{2} \\
& +\frac{\left(v_{n m o}^{2}(1+2 \eta) \sin ^{2} \theta+v_{0}^{2} \cos ^{2} \theta\right)}{c(\tau)}\left(\frac{\partial \tau}{\partial z}\right)^{2}=1,
\end{aligned}
$$

where

$$
\begin{aligned}
c(\tau)= & +2 \eta v_{n m o}^{2} v_{0}^{2}\left(\cos \theta \frac{\partial \tau}{\partial x}+\sin \theta \frac{\partial \tau}{\partial z}\right)^{2} \\
& \times\left(-\sin \theta \frac{\partial \tau}{\partial x}+\cos \theta \frac{\partial \tau}{\partial z}\right)^{2}-2\left(v_{n m o}^{2}(1+2 \eta)-v_{0}^{2}\right) \\
& \times \sin \theta \cos \theta\left(\frac{\partial \tau}{\partial x}\right)\left(\frac{\partial \tau}{\partial z}\right) .
\end{aligned}
$$

By comparing the TTI eikonal equation (5) with the eikonal equation for the much simpler VEA medium:

$v_{\text {nmo }}^{2}\left(\frac{\partial \tau}{\partial x}\right)^{2}+v_{0}^{2}\left(\frac{\partial \tau}{\partial z}\right)^{2}=1$,

we obtain an effective VEA model that captures the kinematic effects of wave propagation in TTI medium. The effective velocities for a VEA medium, embedding the effects due to $\eta$ and $\theta$, are given as:

$$
\begin{gathered}
v_{n m o, e f f}(x, z, s)=\sqrt{\frac{v_{n m o}^{2}(1+2 \eta) \cos ^{2} \theta+v_{0}^{2} \sin ^{2} \theta}{c(\tau)}}, \\
v_{0, e f f}(x, z, s)=\sqrt{\frac{v_{n m o}^{2}(1+2 \eta) \sin ^{2} \theta+v_{0}^{2} \cos ^{2} \theta}{c(\tau)}},
\end{gathered}
$$

where $v_{\text {nmo,eff }}(x, z, s)$ and $v_{0, e f f}(x, z, s)$ are, respectively, the effective P-wave vertical and NMO velocities at location $(x, z)$ for a source position $s$.

Function $c(\tau)$ relies on the solution for the TTI eikonal equation and is evaluated by using an iterative fast-sweepingbased solver (Waheed, Yarman, and Flagg 2014). The solver computes $c(\tau)$ as a by-product at the final iteration. These 


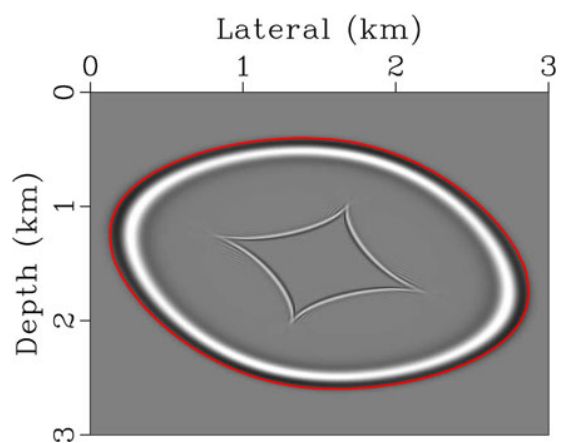

Figure 1 Wavefield snapshot at 0.6 second for the homogeneous TTI model using conventional TTI wavefield extrapolator for a source located at $(1.5 \mathrm{~km}, 1.5 \mathrm{~km})$. Also overlaid is the corresponding traveltime contour obtained by solving the TTI eikonal equation.

effective velocities are functions of source location and will change as the source position varies.

We can then use these effective velocities in the VEA extrapolation operator to obtain a wavefield solution that correctly captures kinematic aspects of the TTI model. The resulting wave equation for a VEA medium using effective velocities is given as:

$\frac{\partial^{2} P}{\partial t^{2}}=v_{n m o, e f f}^{2} \frac{\partial^{2} P}{\partial x^{2}}+v_{0, e f f}^{2} \frac{\partial^{2} P}{\partial z^{2}}$.

\section{Effective tilted elliptically anisotropic model}

By setting $\eta=0$ in the TTI wave equation (2), we obtain the wave equation for an elliptically anisotropic medium with tilted axis of symmetry (TEA) to be:

$$
\begin{aligned}
\frac{\partial^{2} P}{\partial t^{2}}= & \left(v_{n m o}^{2} \frac{\partial^{2} P}{\partial x^{2}}+v_{0}^{2} \frac{\partial^{2} P}{\partial z^{2}}\right) \cos ^{2} \theta+\left(v_{0}^{2} \frac{\partial^{2} P}{\partial x^{2}}+v_{n m o}^{2} \frac{\partial^{2} P}{\partial z^{2}}\right) \\
& \times \sin ^{2} \theta+\left(v_{n m o}^{2}-v_{0}^{2}\right) \frac{\partial^{2} P}{\partial x \partial z} \sin 2 \theta .
\end{aligned}
$$

Using a similar analysis, it is also possible to obtain an effective TEA model for wavefield extrapolation in TTI media. Here, only the influence of $\eta$ will be embedded in the velocity models, whereas the original tilt model of the TTI medium will be used in the effective model. In a similar manner as above, we compare the TTI and TEA eikonal equations and derive expressions for the effective TEA medium to be:

$$
\begin{aligned}
\widetilde{v}_{\text {nmo }, e f f}(x, z, s) & =v_{\text {nmo }} \sqrt{\frac{1+2 \eta}{\widetilde{c}(\tau)}}, \\
\widetilde{v}_{0, e f f}(x, z, s) & =\frac{v_{0}}{\sqrt{\tilde{c}(\tau)}},
\end{aligned}
$$

where

$$
\begin{aligned}
\widetilde{c}(\tau)= & 1+2 \eta v_{n m o}^{2} v_{0}^{2}\left(\cos \theta \frac{\partial \tau}{\partial x}+\sin \theta \frac{\partial \tau}{\partial z}\right)^{2} \\
& \times\left(-\sin \theta \frac{\partial \tau}{\partial x}+\cos \theta \frac{\partial \tau}{\partial z}\right)^{2} .
\end{aligned}
$$

In equation (11), $\widetilde{v}_{m m o, e f f}(x, z, s)$ and $\widetilde{v}_{0, e f f}(x, z, s)$ denote, respectively, the effective NMO and P-wave velocities along the local symmetry direction at location $(x, z)$ for a source $s$.

We can then solve the TEA wave equation using effective velocities given by equation (11) and obtain approximate wavefield solution for a TTI model. The resulting wave equation for effective TEA medium would then be given as:

$$
\begin{aligned}
\frac{\partial^{2} P}{\partial t^{2}}= & \left(\widetilde{v}_{n m o, e f f}^{2} \frac{\partial^{2} P}{\partial x^{2}}+\widetilde{v}_{0, e f f}^{2} \frac{\partial^{2} P}{\partial z^{2}}\right) \cos ^{2} \theta \\
& +\left(\widetilde{v}_{0, e f f}^{2} \frac{\partial^{2} P}{\partial x^{2}}+\widetilde{v}_{n m o, e f f}^{2} \frac{\partial^{2} P}{\partial z^{2}}\right) \sin ^{2} \theta \\
& +\left(\widetilde{v}_{n m o, e f f}^{2}-\widetilde{v}_{0, e f f}^{2}\right) \frac{\partial^{2} P}{\partial x \partial z} \sin 2 \theta .
\end{aligned}
$$

The TEA wavefield extrapolation operator is relatively simpler and computationally cheaper to solve than the one for the TTI media by a factor of about two.

We considered here a 2D TTI medium for simplicity of illustration. Expressions for effective velocities approximating wavefield for a 3D TTI medium are given in Appendix.

\section{ALGORITHM}

In this section, we describe steps needed to compute an approximate wavefield solution for TTI medium using effective elliptically anisotropic models.

In order to obtain an effective VEA model that embeds kinematic effects due to $\eta$ and $\theta$ into the effective velocities, first we solve the TTI eikonal equation (4). We use an iterative fast-sweeping-based eikonal solver. Details of the eikonal solver can be found in Waheed et al. (2014). As a by-product, the algorithm yields the right-hand-side function $c(\tau)$ at the final iteration, which is then used to compute effective velocities using equation (8). Once effective velocities are obtained, we then use the VEA wave equation with these velocities to compute an approximate wavefield solution for TTI medium (see equation (9)). A summary of these steps is given in Algorithm (1). 
Figure 2 Wavefield snapshots at 0.6 second for the homogeneous TTI model using: (a) TEA wavefield extrapolator ignoring the $\eta$ parameter and (b) TEA wavefield extrapolator employing effective TEA model. Also overlaid are the corresponding travel-time contours obtained by solving the TTI eikonal equation. The source is located at $(1.5 \mathrm{~km}, 1.5 \mathrm{~km})$.

Figure 3 Difference between velocities (in $\mathrm{km} / \mathrm{s}$ ) for the effective TEA model and the original TTI model: (a) $\widetilde{v}_{0, e f f}-v_{0}$ and (b) $\widetilde{v}_{\text {nmo,eff }}-v_{\text {nmo }}$ for a source located at $(1.5 \mathrm{~km}, 1.5 \mathrm{~km})$.

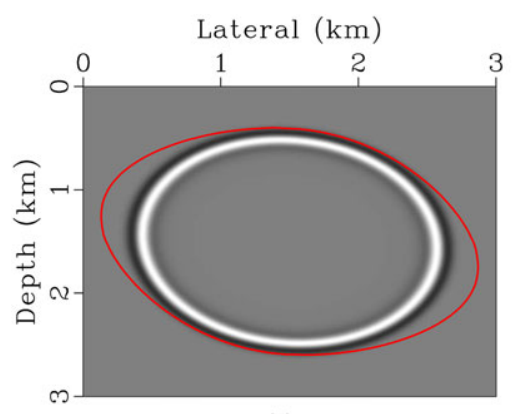

(a)

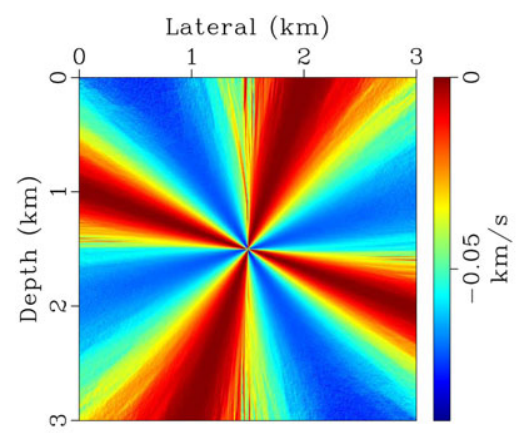

(a)

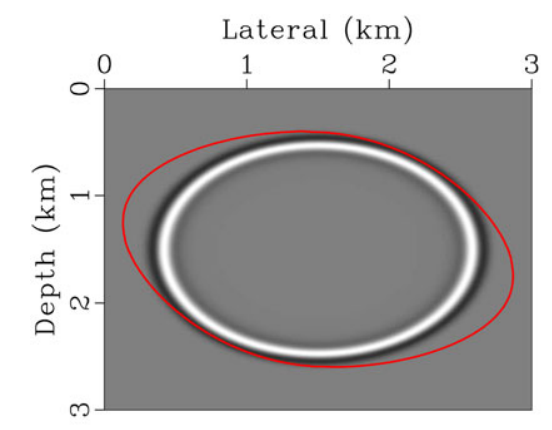

(a)

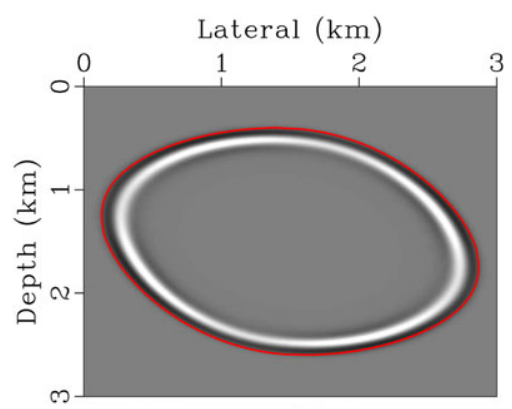

(b)

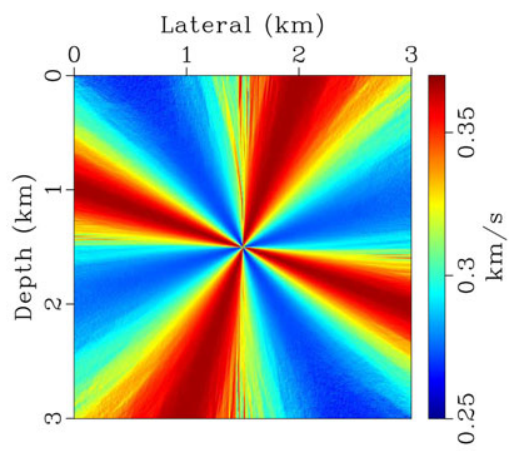

(b)

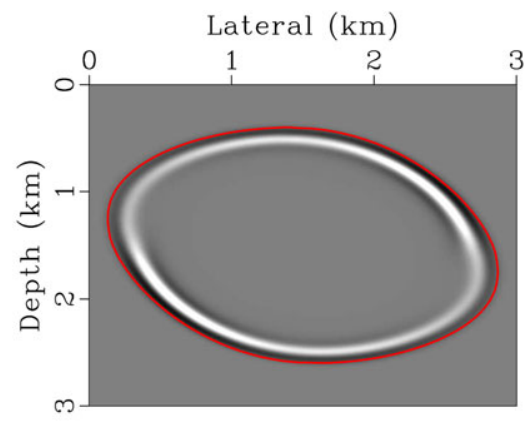

(b)
Figure 4 Wavefield snapshots at 0.6 second for the homogeneous TTI model using: (a) VEA wavefield extrapolator ignoring the $\eta$ and $\theta$ parameters and (b) VEA wavefield extrapolator employing effective VEA model. Also overlaid are the corresponding travel-time contours obtained by solving the TTI eikonal equation. The source is located at $(1.5 \mathrm{~km}, 1.5 \mathrm{~km})$.

Algorithm 1. Efficient wavefield extrapolation in TTI media using an effective VEA model

1. Compute the TTI travel-time solution $\tau$ using equation (4).

2. Evaluate the right-hand-side function $c(\tau)$ using equation (6).

3. Compute effective velocities $v_{0, \text { eff }}$ and $v_{\text {nmo,eff }}$ from equation (8).

4. Solve for approximate TTI wavefield solution using equation (9).

In a similar manner we can obtain an effective TEA model by computing effective velocities from equation (11) and then solve for the approximate wavefield using equation (13).

\section{NUMERICAL TESTS}

In this section, we test accuracy of effective VEA and effective TEA models in approximating wavefield solution 


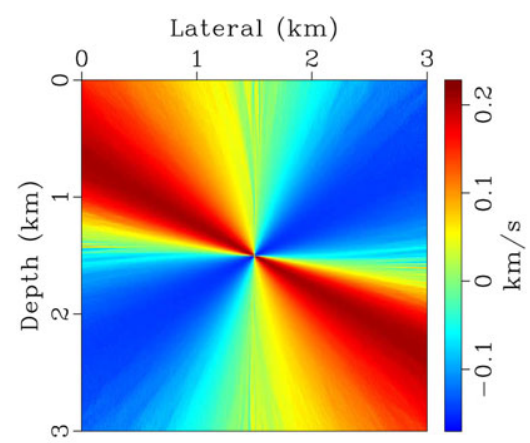

(a)

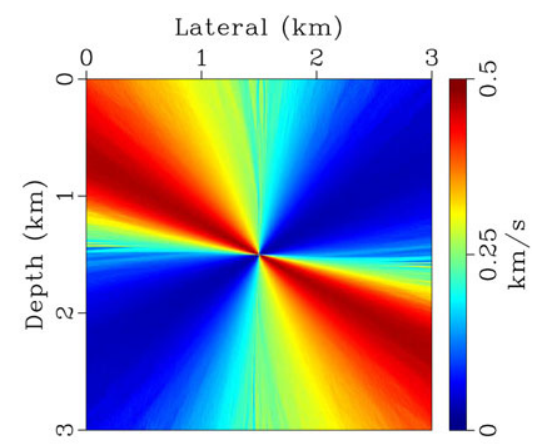

(b)
Figure 5 Difference between velocities (in $\mathrm{km} / \mathrm{s}$ ) for the effective VEA model and the original TTI model: (a) $v_{0, e f f}-v_{0}$ and (b) $v_{\text {nmo,eff }}-v_{\text {nmo }}$ for a source located at $(1.5 \mathrm{~km}, 1.5 \mathrm{~km})$. for TTI media. We consider a homogeneous TTI model, a strongly anisotropic layered TTI model, and part of the BP TTI model (Shah 2007). The homogeneous TTI model will help us understand accuracy behaviour of the approximation, easily indicating regions where the approximation yields sufficient accuracy and where it does not. The layered TTI model introduces reflected waves into the seismic wavefield. Reconstructing secondary events in the presence of strong anisotropy constitutes a stiff test for the approximation. It will aid in understanding limitations of the proposed algorithm. Finally, the BP TTI model will help us assess performance of the method under practical scenario. The considered portion of the BP model is particularly revealing due to relatively large $\eta$ values and sharp variation in tilt angles.

\section{Example 1: Homogeneous TTI model}

We consider a $3 \mathrm{~km} \times 3 \mathrm{~km}$ homogeneous TTI model with parameters: $v_{0}=1.8 \mathrm{~km} / \mathrm{s}, v_{n m o}=2 \mathrm{~km} / \mathrm{s}, \eta=0.2$, and $\theta=20^{\circ}$. For a source located at $(1.5 \mathrm{~km}, 1.5 \mathrm{~km})$, Fig. 1 shows wavefield snapshot for the model at 0.6 second using a secondorder in time and fourth-order in space finite-difference-based TTI wavefield extrapolator. The grid spacing used in both directions is $5 \mathrm{~m}$, whereas peak frequency of the Ricker wavelet is $10 \mathrm{~Hz}$. In addition, we overlay the corresponding TTI eikonal solution (in red). As expected, the first break of the wavefield correctly matches the TTI eikonal solution. The star-shaped structure at the centre is the shear-wave artefact.

Due to huge computational cost associated with the TTI wavefield extrapolator, we use a relatively cheaper TEA wavefield solver, at first by just ignoring the $\eta$ parameter in the TTI model. The inaccuracy due to neglecting $\eta$ is depicted in Fig. 2(a). The plot shows the wavefield solution using a TEA wavefield solver overlaid by the TTI eikonal solution, for comparison, at 0.6 second. Due to large errors, we embed kinematic effects due to $\eta$ into an effective TEA model and obtain effective velocities $\left(\widetilde{v}_{0, e f f}, \widetilde{v}_{n m o, e f f}\right)$ using equation (11). The difference between the obtained effective velocities and the original velocities is shown in Fig. 3. Figure 2(b) shows wavefield snapshot of the wavefield solution corrected using the effective TEA model. Notice that the fastest arriving wave correctly matches the TTI eikonal solution (in red) despite using a cheaper wave extrapolation operator. In addition, the wavefield solution is free from any shear-wave noise.

Next we consider an even simpler VEA wavefield extrapolator for the homogeneous TTI model. At first by ignoring the $\eta$ and $\theta$ parameters, Fig. 4(a) shows the wavefield snapshot obtained using a VEA wave extrapolator at 0.6 second. Again we observe large errors by comparing it with the corresponding TTI eikonal solution. Therefore, we obtain effective velocities $\left(v_{0, e f f}, v_{n m o, e f f}\right)$ for the VEA model using equation (8). The difference between the effective velocities and original velocities is shown in Fig. 5. We can observe that the first arrival of the corrected wavefield, in Fig. 4(b), matches the TTI eikonal solution. Again the effective model-based wavefield solution does not contain shear-wave artefacts.

In order to compare accuracy properties of the different wavefield solutions, we compare wavefield profiles in Fig. 6. The profiles shown in Fig. 6(a) and (b) correspond to depth slices ( $z=1 \mathrm{~km}$, and $z=5 \mathrm{~km}$, respectively) from the wavefield snapshot at 0.6 second. Similarly, Fig. 6(c) and (d) shows vertical slices ( $x=1 \mathrm{~km}$, and $x=5 \mathrm{~km}$, respectively) from the wavefield snapshot at 0.6 second. Fig. $6(\mathrm{e})$ and (f) shows the wavefields recorded in time at $(\mathrm{x}, \mathrm{z})=(0.75 \mathrm{~km}, 1 \mathrm{~km})$, and $(\mathrm{x}, \mathrm{z})=(1.2 \mathrm{~km}, 1.5 \mathrm{~km})$, respectively. In this figure and the following, wavefield profiles obtained using the conventional TTI wave extrapolator is shown in blue, whereas wavefield profiles from the effective TEA and the effective VEA-based extrapolators are plotted in red and green, respectively. 


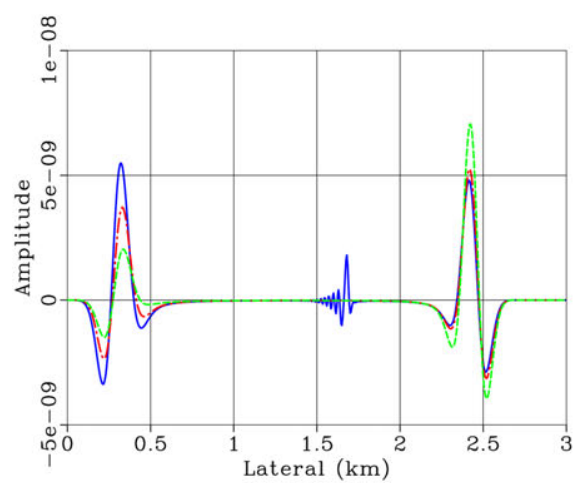

(a)

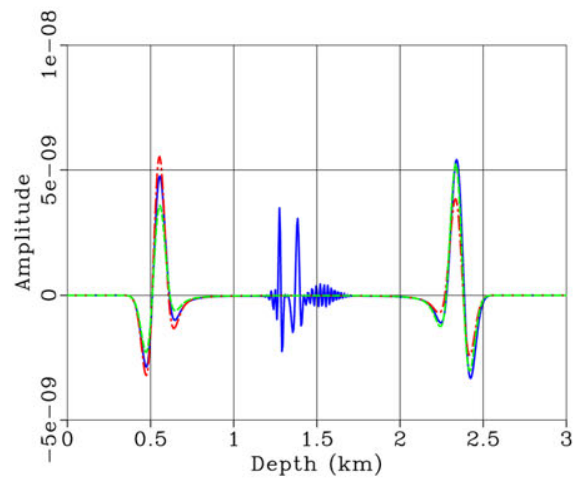

(c)

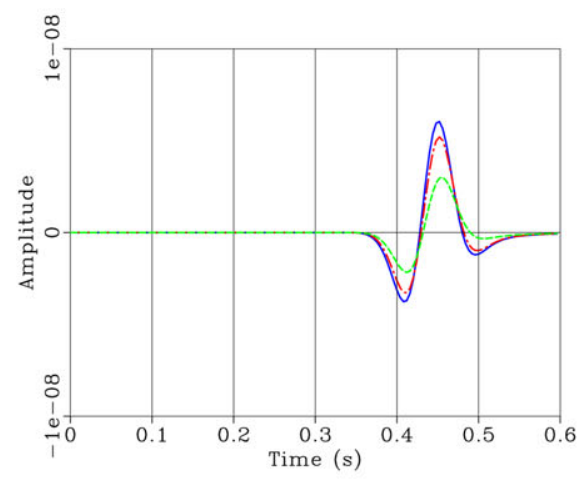

(e)

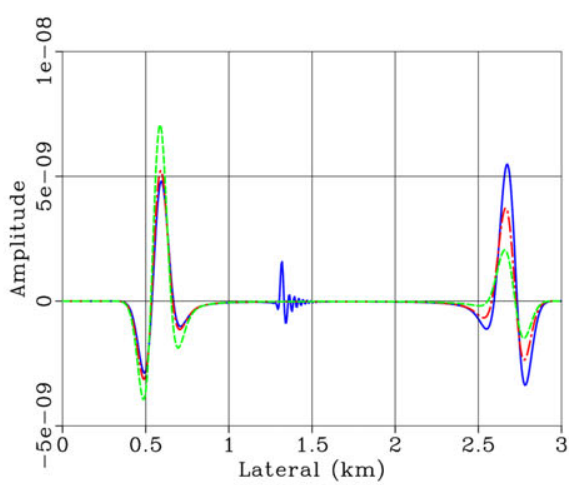

(b)

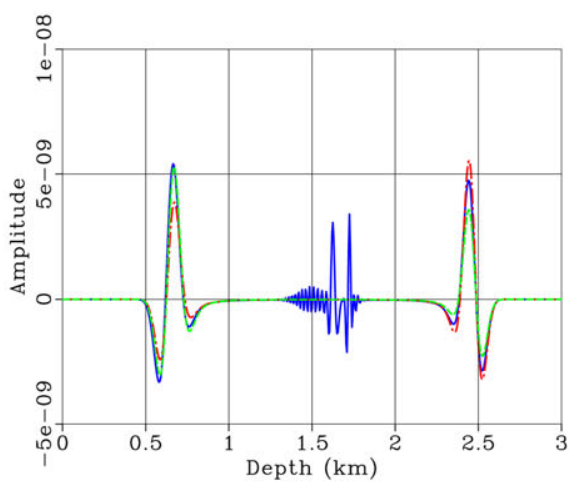

(d)

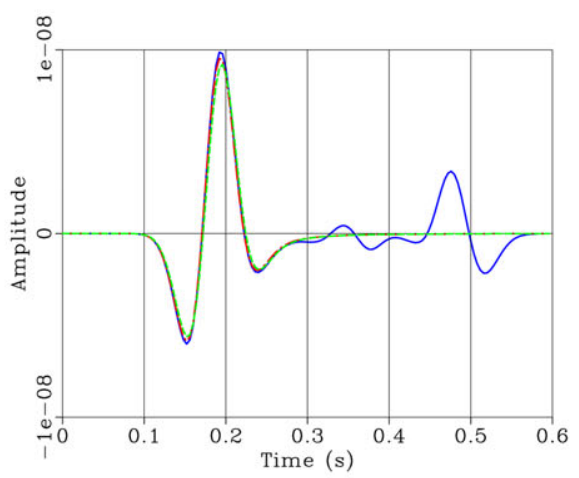

(f)

Figure 6 Wavefield profiles for the homogeneous TTI model using TTI wavefield extrapolator (blue) compared with profiles from effective TEA wave extrapolator (red) and effective VEA extrapolator (green) at: (a) $t=0.6$ second, $z=1 \mathrm{~km}$; (b) $t=0.6 \mathrm{~second}, z=2 \mathrm{~km}$; (c) $t=0.6$ second, $x=1 \mathrm{~km}$; (d) $t=0.6$ second, $x=2 \mathrm{~km}$; (e) $x=0.75 \mathrm{~km}, z=1 \mathrm{~km}$; and (f) $x=1.2 \mathrm{~km}, z=1.5 \mathrm{~km}$. Peak frequency of the Ricker wavelet used is $10 \mathrm{~Hz}$.

From the profiles in Fig. 6 we observe that both the elliptic wave extrapolators are able to correctly match the phase information of the TTI wavefield. However, the effective TEA-based wave extrapolator yields a better amplitude match compared with the effective VEA-based extrapolator. The additional events appearing only in the
TTI wavefield profiles (blue) are the unwanted shear-wave artefacts.

To compare accuracy properties of effective elliptic models in different frequency bands, we repeat the experiment using Ricker wavelet with a peak frequency of $30 \mathrm{~Hz}$. The wavefield profiles corresponding to Fig. 6 are plotted in 


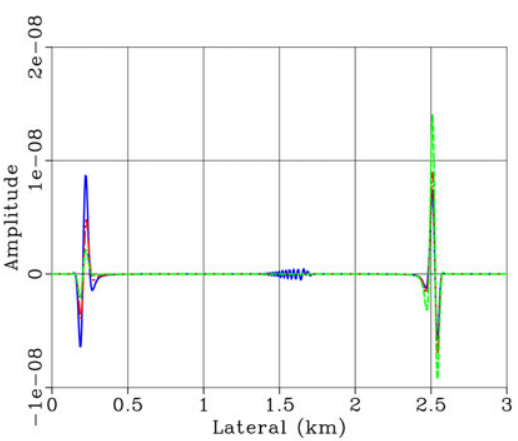

(a)

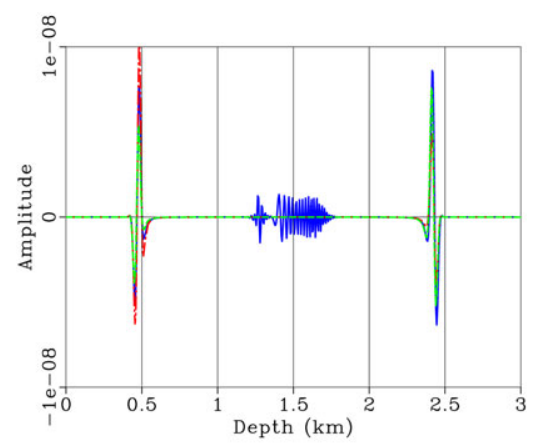

(c)

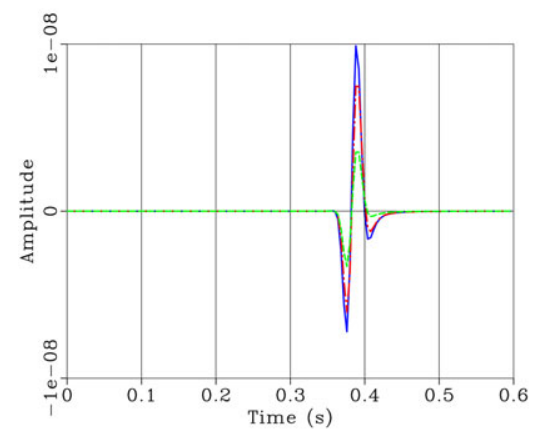

(e)

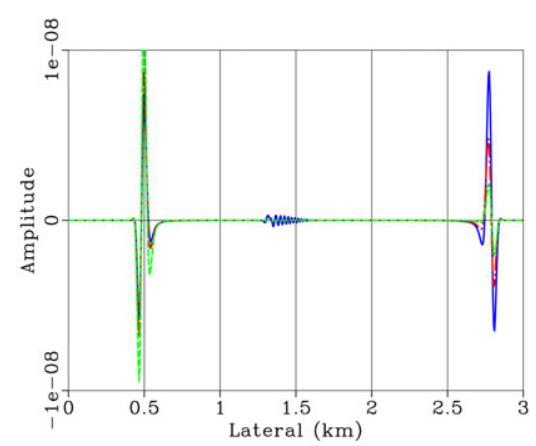

(b)

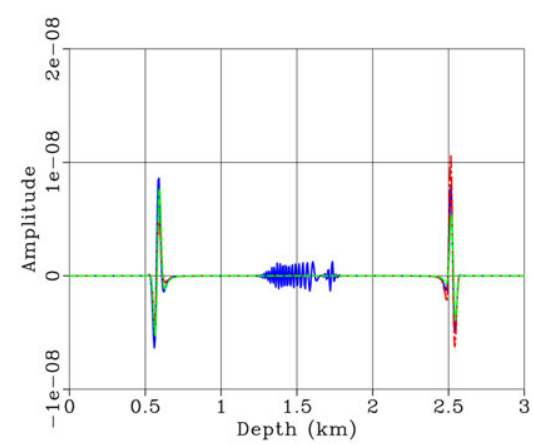

(d)

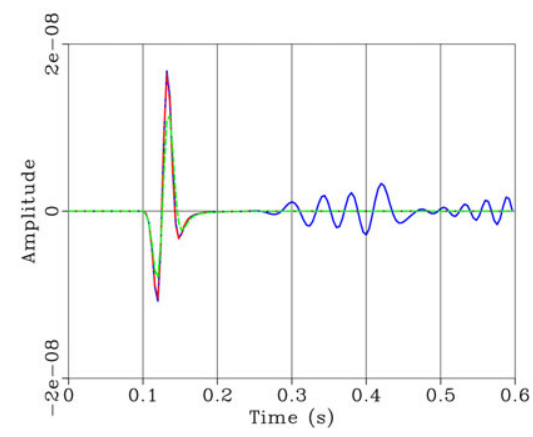

(f)
Figure 7 Wavefield profiles for the homogeneous TTI model using TTI wavefield extrapolator (blue) compared with profiles from effective TEA wave extrapolator (red) and effective VEA extrapolator (green) at: (a) $t=0.6$ second, $z=1 \mathrm{~km}$; (b) $t=0.6$ second, $z=2 \mathrm{~km}$; (c) $t=0.6 \mathrm{sec}-$ ond, $x=1 \mathrm{~km}$; (d) $t=0.6$ second, $x=2 \mathrm{~km}$; (e) $x=0.75 \mathrm{~km}, z=1 \mathrm{~km}$; and (f) $x=1.2 \mathrm{~km}$, $z=1.5 \mathrm{~km}$. Peak frequency of the Ricker wavelet used is $30 \mathrm{~Hz}$.
Table 1 Medium parameters for a three-layer TTI model. Each layer has dimensions $2 \mathrm{~km} \times 6 \mathrm{~km}$. Layer 1 refers to the top layer, layer 2 is the middle layer, whereas layer 3 is the bottom layer.

\begin{tabular}{llll}
\hline & Layer 1 & Layer 2 & Layer 3 \\
\hline$v_{0}$ & $1.5 \mathrm{~km} / \mathrm{s}$ & $1.8 \mathrm{~km} / \mathrm{s}$ & $2.1 \mathrm{~km} / \mathrm{s}$ \\
$v_{n m o}$ & $1.64 \mathrm{~km} / \mathrm{s}$ & $2.13 \mathrm{~km} / \mathrm{s}$ & $2.7 \mathrm{~km} / \mathrm{s}$ \\
$\eta$ & 0.05 & 0.15 & 0.25 \\
$\theta$ & $20^{\circ}$ & $10^{\circ}$ & $-30^{\circ}$ \\
\hline
\end{tabular}

Fig. 7. The amplitude and phase properties of the effective models bear great resemblance in both frequency bands. The only effect noticeable is the slight distortion in the wavelet for effective model-based solutions. This is due to change in velocity compared to the original TTI model. This distortion becomes prominent as the frequency decreases. Even though the difference is barely noticeable, we can observe this by comparing wavefield profiles for the effective VEA case (green).

For the following tests, we will consider Ricker wavelet with peak frequency of $10 \mathrm{~Hz}$ only.

\section{Example 2: Layered TTI model}

To better understand accuracy properties of effective elliptic models in approximating secondary arrivals, we consider a 


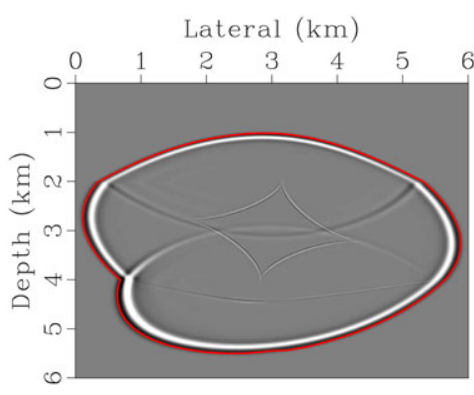

(a)

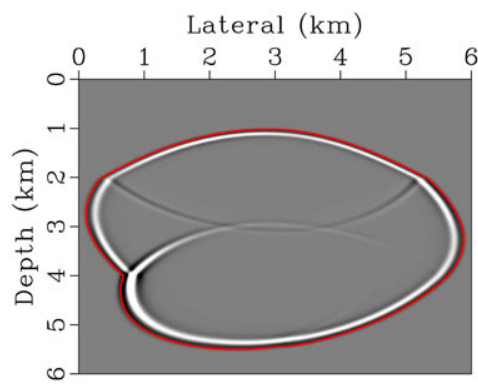

(b)

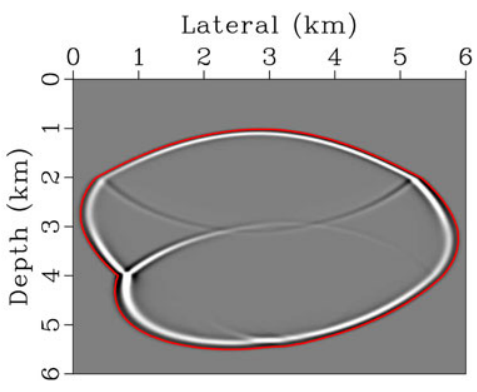

(c)

Figure 8 Wavefield snapshots for the layered TTI model at 1.2 seconds using (a) TTI wavefield extrapolator, (b) TEA wavefield extrapolator employing effective TEA model, and (c) VEA wavefield extrapolator employing effective VEA model. Also overlaid are the corresponding travel-time contours obtained by solving the TTI eikonal equation. The source is located at $(3 \mathrm{~km}, 3 \mathrm{~km})$.

Figure 9 Difference between velocities (in $\mathrm{km} / \mathrm{s}$ ) for the effective models and the original TTI model: (a) $\widetilde{v}_{0, e f f}-v_{0}$, (b) $\widetilde{v}_{\text {nmo,eff }}-v_{n m o}$, (c) $v_{0, e f f}-v_{0}$, and (d) $v_{\text {nmo,eff }}-v_{\text {nmo }}$ for a source located at $(3 \mathrm{~km}, 3 \mathrm{~km})$.

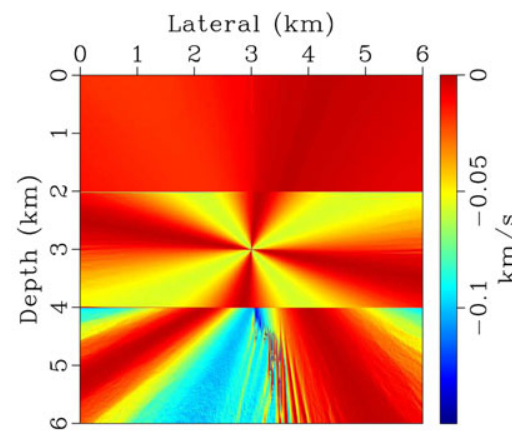

(a)

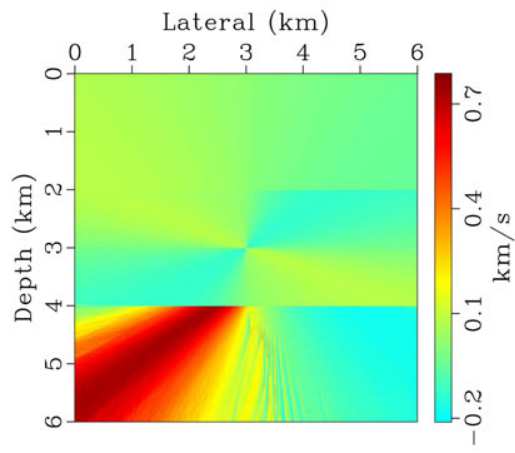

(c)

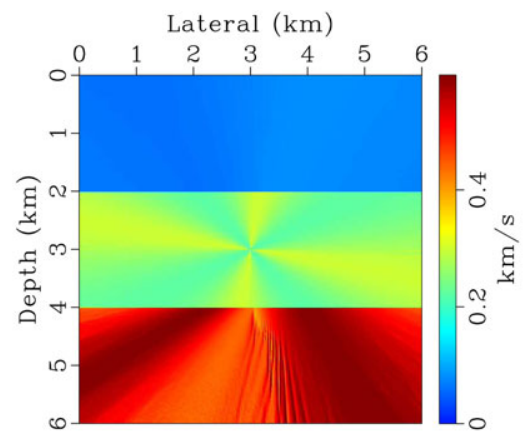

(b)

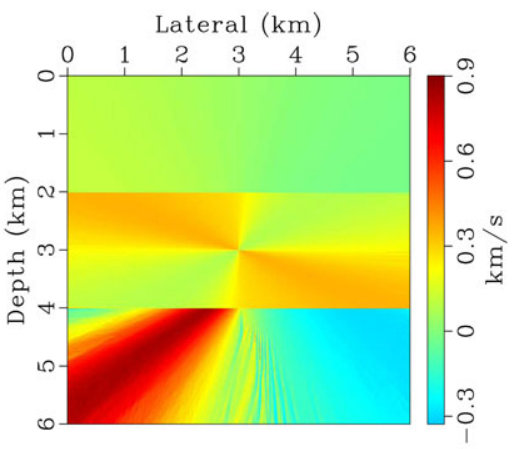

(d) layered TTI model. Furthermore, to observe the behaviour of the approximation in harsh geological settings, we consider a strongly anisotropic medium with strength of anisotropy increasing as we go further deep. The model parameters are given in Table 1. Each layer is flat and has a dimension of $2 \mathrm{~km} \times 6 \mathrm{~km}$. A grid spacing of $10 \mathrm{~m}$ is used along both axes.

For a source located at $(3 \mathrm{~km}, 3 \mathrm{~km})$, Fig. $8(\mathrm{a})$ shows wavefield snapshot for the model at 1.2 seconds using the conventional TTI wavefield extrapolator. As expected, the corresponding eikonal solution (in red) correctly matches the first arriving wave. Next, we plot the corresponding wavefield snapshot obtained using the effective TEA model in Fig. 8(b). The effective TEA-based wavefield solution also matches the TTI eikonal solution. The difference between the original and the effective velocities is shown in Fig. 9(a) and (b). Notice that, due to the presence of strong anisotropy in the original TTI model, velocity differences go up to almost $0.6 \mathrm{~km} / \mathrm{s}$ in the bottom layer.

Figure $8(\mathrm{c})$ plots the wavefield snapshot at 1.2 seconds using VEA wave extrapolator employing effective VEA model. It can be easily observed that the first arrival of the wavefield 


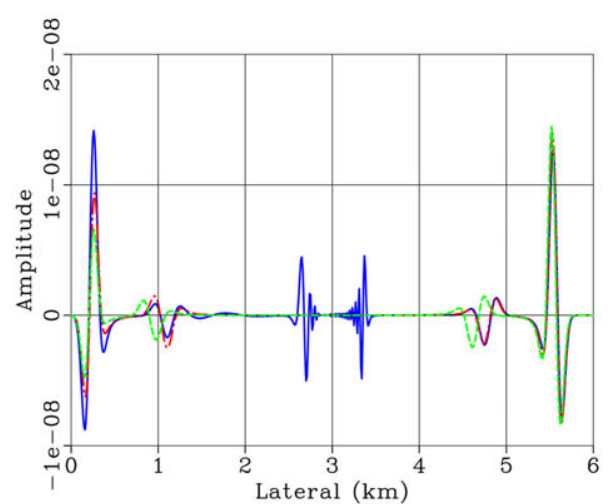

(a)

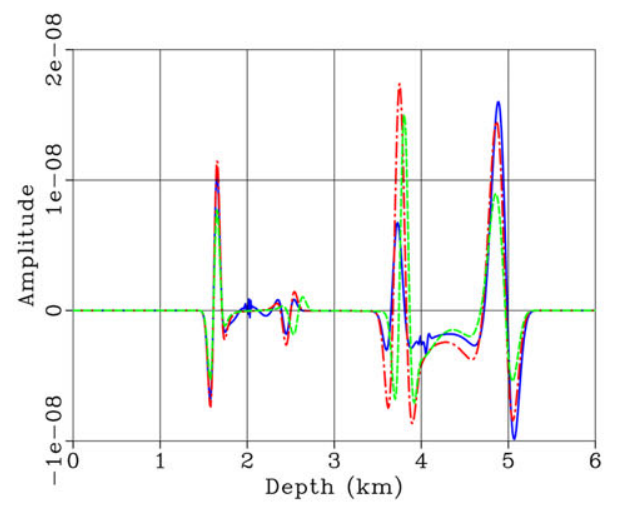

(c)

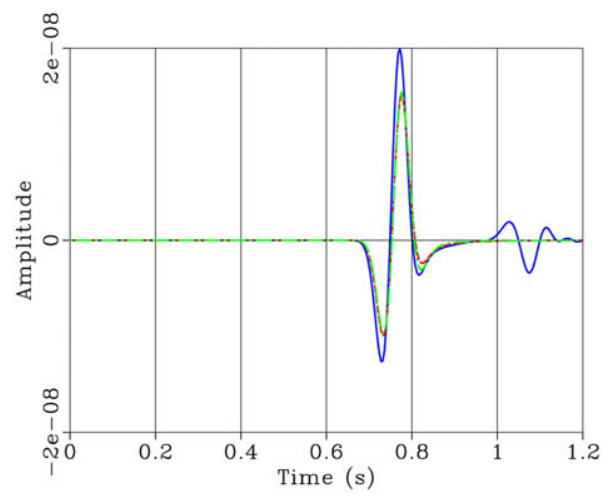

(e)

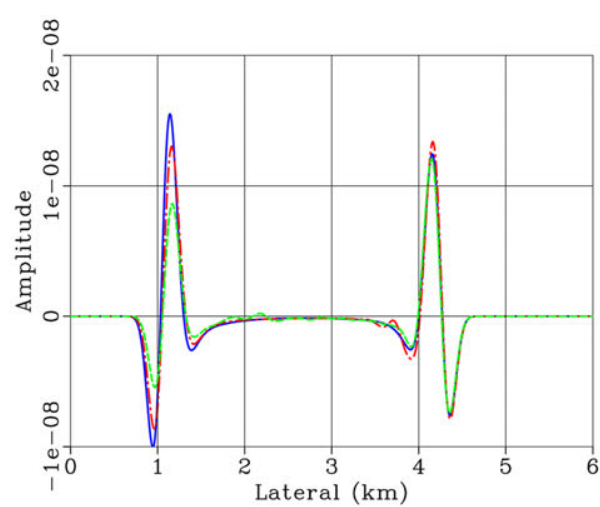

(b)

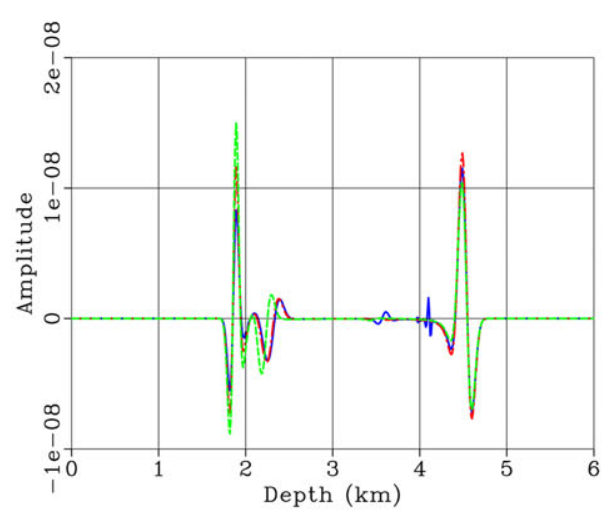

(d)

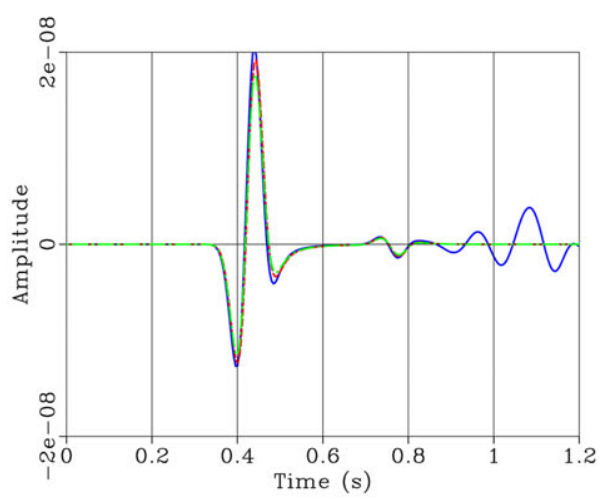

(f)

Figure 10 Wavefield profiles for the layered TTI model using TTI wavefield extrapolator (blue) compared with profiles from effective TEA wave extrapolator (red) and effective VEA extrapolator (green) at: (a) $t=1.2$ seconds, $z=2.5 \mathrm{~km}$; (b) $t=1.2 \mathrm{~seconds}, z=5 \mathrm{~km}$; (c) $t=1.2$ seconds, $x=1 \mathrm{~km}$; (d) $t=1.2$ seconds, $x=5 \mathrm{~km}$; (e) $x=2.5 \mathrm{~km}, z=4.3 \mathrm{~km}$; and (f) $x=3 \mathrm{~km}, z=2.5 \mathrm{~km}$.

solution matches the corresponding TTI eikonal solution. The difference between the original and the effective VEA velocities is shown in Fig. 9(c) and (d). Due to large tilt values and strong anisotropy in the original TTI model, the velocity difference reaches up to $0.9 \mathrm{~km} / \mathrm{s}$ in the bottom layer.
To better understand accuracy of different extrapolators, we plot wavefield profiles in Fig. 10. We observe that both effective models yield a perfect kinematic match for the first arriving wave. However, accuracy of amplitudes for the first arrivals is better for the effective TEA model (red) compared 
Figure 11 A portion of the BP TTI model depicting: (a) the symmetry axis velocity, (b) the NMO velocity, (c) the $\eta$ parameter, and (d) the tilt.

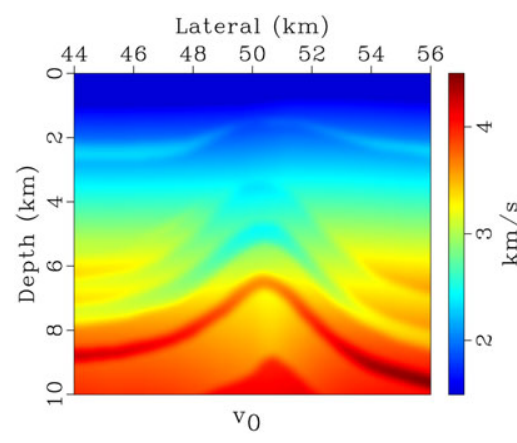

(a)

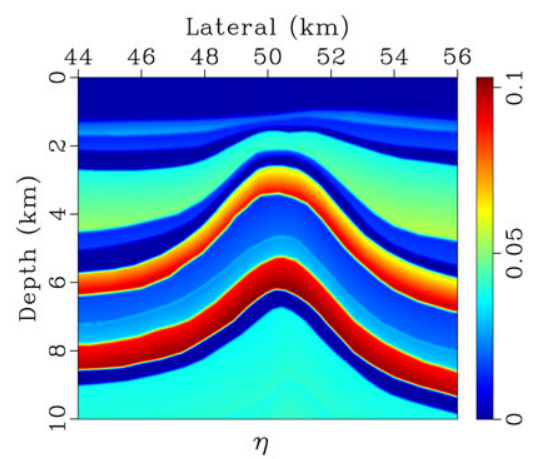

(c)

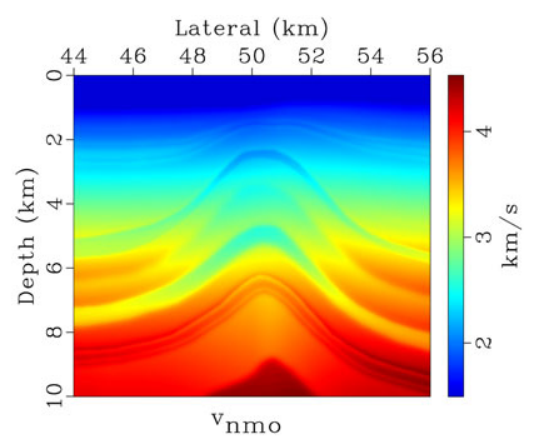

(b)

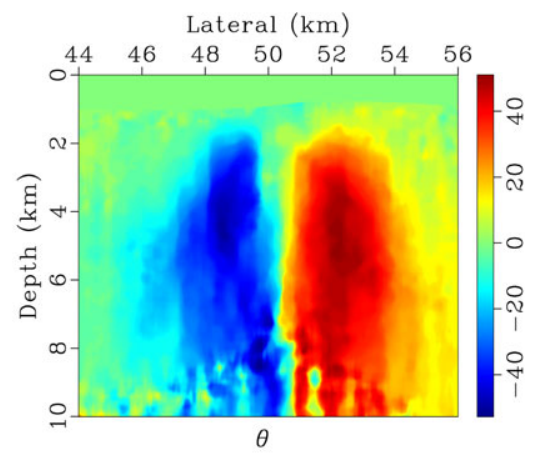

(d)

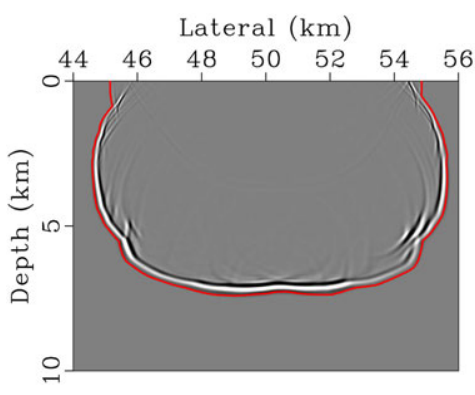

(a)

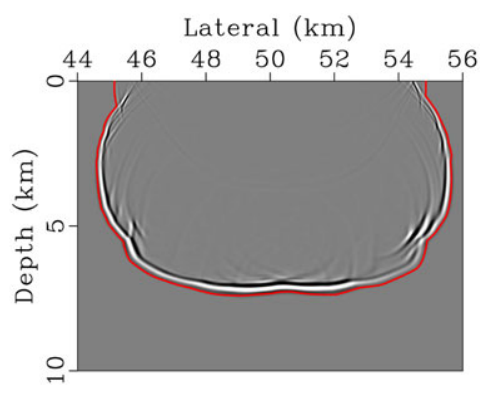

(b)

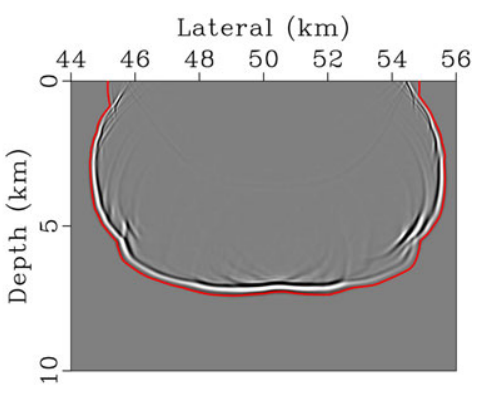

(c)

Figure 12 Wavefield snapshots for the BP TTI model at 3.25 seconds using: (a) TTI wavefield extrapolator, (b) TEA wavefield extrapolator employing effective TEA model, and (c) VEA wavefield extrapolator employing effective VEA model. Also overlaid are the corresponding travel-time contours obtained by solving the TTI eikonal equation. The source is located at $(50 \mathrm{~km}, 0 \mathrm{~km})$.

with that for the effective VEA model (green). Despite fitting kinematics only using the eikonal solution, the effective TEA model also matches phase information of reflected events with reasonable accuracy. However, the effective VEA model yields a poor kinematic approximation for the secondary arrivals. This is due to the fact that the VEA model embeds information from both $\eta$ and $\theta$ fields. Since these parameters are significantly large, the eikonal-based fitting ensures that the phase of the first arrival is correctly matched, leaving the secondary arrivals with reduced accuracy.
Of note is the event originating in the conventional TTI wavefield solution (blue) at about 1 second, shown in Fig. $10(\mathrm{e})$. This event is due to the wave transmitted from the interface between layers 2 and 3 after getting reflected from the interface formed by layers 1 and 2 . The event is absent from the effective model-based solutions. Due to large differences in the original and effective velocities, impedance change in the effective model between different layers is much lesser than in the original TTI model. Therefore, energy due to such events may die out earlier in the effective model. The remaining 


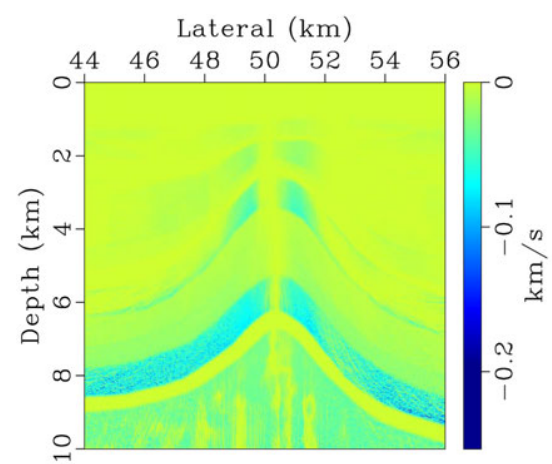

(a)

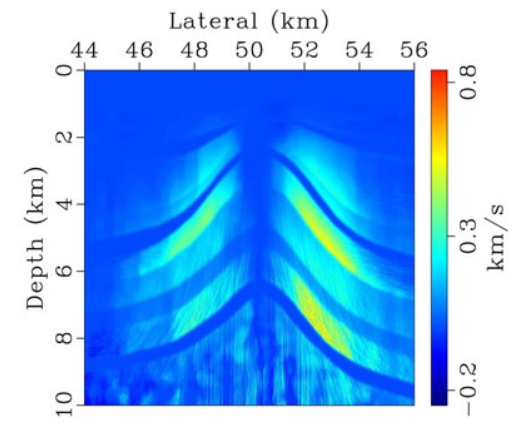

(c)

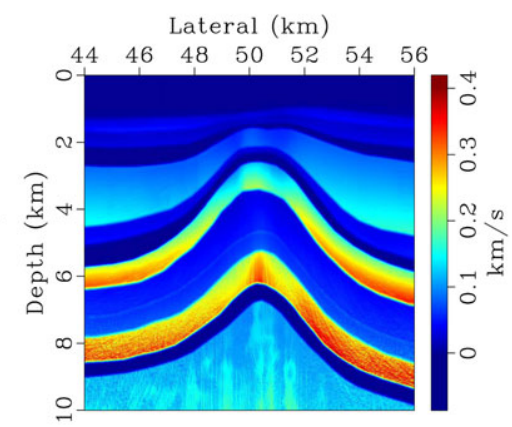

(b)

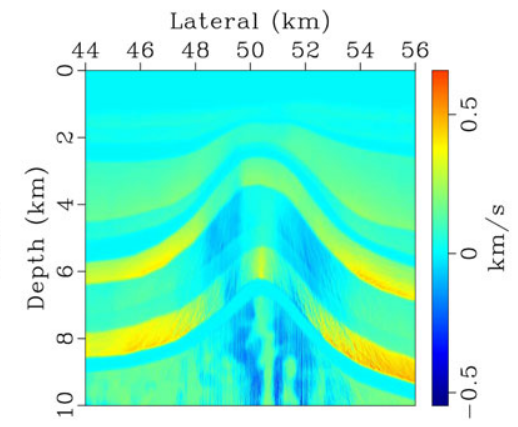

(d)
Figure 13 Difference between velocities (in $\mathrm{km} / \mathrm{s}$ ) for the effective models and the original TTI model: (a) $\widetilde{v}_{0, e f f}-v_{0}$, (b) $\widetilde{v}_{n m o, e f f}-v_{n m o}$, (c) $v_{0, e f f}-v_{0}$, and (d) $v_{n m o, e f f}-v_{n m o}$ for a source located at $(50 \mathrm{~km}, 0 \mathrm{~km})$. events in the wavefield profiles not captured by the effective model-based solution are the unwanted shear-wave artefacts.

\section{Example 3: BP TTI model}

Finally, we consider part of the BP TTI model shown in Fig. 11. A grid spacing of $6.25 \mathrm{~m}$ is used in both dimensions. We consider a source at the surface, located at $(50 \mathrm{~km}, 0 \mathrm{~km})$ and propagate wavefields using Ricker wavelet with peak frequency of $10 \mathrm{~Hz}$. The wavefield snapshot at 3.25 seconds using the computationally expensive TTI wavefield extrapolator is shown in Fig. 12(a). As expected, the first arriving wave correctly matches the corresponding TTI eikonal solution.

Fig. 12(b) and (c) shows wavefield snapshots obtained using the effective TEA and the effective VEA-based extrapolators, respectively. In both cases, the first arriving wave correctly matches the TTI eikonal solution. The difference between the original and the effective velocities is shown in Fig. 13.

The wavefield profiles comparing different solutions are plotted in Fig. 14. We observe that the effective TEA-based wave extrapolator yields a nearly perfect match in reconstructing both amplitude and phase information for all events in the TTI wavefield. The extrapolator using effective VEA model yields slightly lesser amplitude accuracy. However, it still matches the phase information of later arrivals with reasonable accuracy.

The wavefield profiles presented above for the three TTI models depict worst case scenarios. Therefore, for most parts of the model, the accuracy is expected to be better than shown by them. Our tests indicate a computational saving of about $60 \%$ and $30 \%$, respectively, for the effective VEA model- and the effective TEA model-based wave extrapolation compared with the conventional TTI wave extrapolation method. The computational savings are expected to be significantly larger for the $3 \mathrm{D}$ case. However, the method is highly reliant on the efficiency of the eikonal solver.

\section{DISCUSSION}

The main motivation behind the proposed methodology is reduction of cost in modelling wavefields for anisotropic media, particularly when it exhibits tilt. Forward modelling usually bottlenecks migration/inversion algorithms as several such modelling operations are needed to obtain a final image of the subsurface. Therefore, the proposed algorithm can help significantly reduce the overall cost of inversion schemes. 
Figure 14 Wavefield profiles for the BP TTI model using TTI wavefield extrapolator (blue) compared with profiles from effective TEA wave extrapolator (red) and effective VEA extrapolator (green) at: (a) $t=3.25$ seconds, $z=4 \mathrm{~km}$; (b) $t=3.25$ seconds, $z=6 \mathrm{~km}$; (c) $t=3.25$ seconds, $x=46 \mathrm{~km}$; (d) $t=3.25$ seconds, $x=54 \mathrm{~km}$; (e) $x=46.5 \mathrm{~km}, z=2.5 \mathrm{~km}$; and (f) $x=54 \mathrm{~km}$, $z=2.5 \mathrm{~km}$.

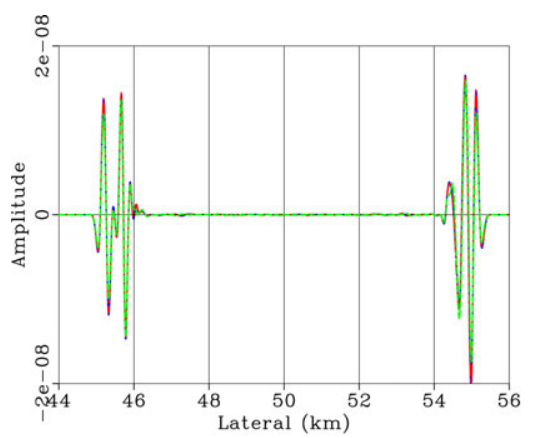

(a)

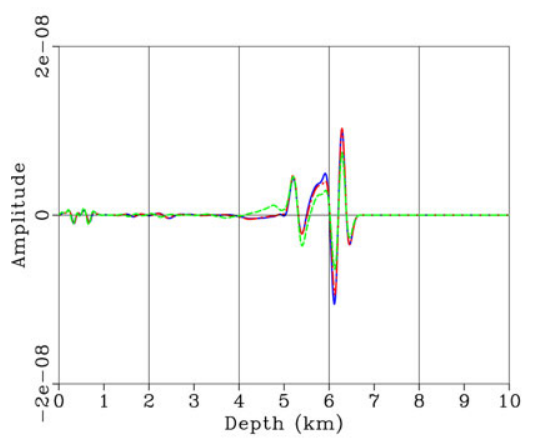

(c)

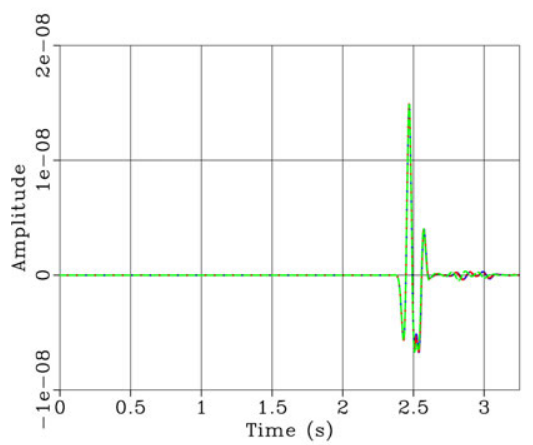

(e)

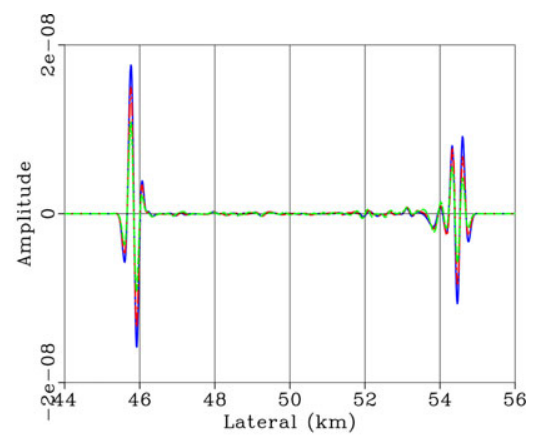

(b)

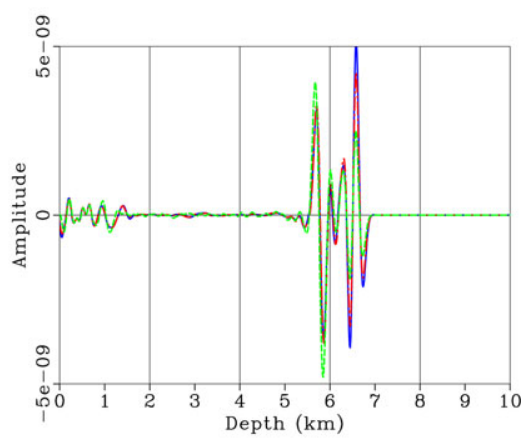

(d)

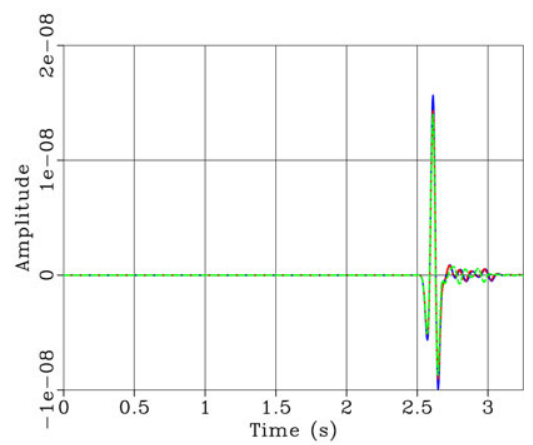

(f)
The formulations developed here allow for an additional control of the cost versus efficiency trade-off. For low to moderately complex media, both approaches yield accurate geometrical features of the wavefields. However, the amplitude accuracy is significantly higher for the effective TEA model. For moderate to highly complex media, the approach presented here can be complemented by obtaining effective density models to obtain a better match of the amplitude features. The effective density and velocities can then be used in a variable density wavefield extrapolator for elliptic media to obtain an accurate approximation of the TTI wavefield (Wang et al. 2014).
In reverse time migration, the receiver wavefield is extrapolated by backward propagating the data recorded at the surface. Since effective velocity models are source position dependent, one needs an effective velocity model for each receiver location, which may be cost prohibitive. Therefore, a more cost-effective approach as proposed by Alkhalifah et al. (2013) is to use delayed shot migration (Zhang et al. 2005; Vigh and Starr 2008).

In delayed shot migration, all sources at the surface are modelled with a delayed time given by a chosen slope with no delay at the origin $x=0$. The same slope is used to sum the receiver wavefields prior to backward extrapolating them. The 
zero lag cross-correlation imaging condition is then used to obtain the image. Several such plane-wave sources, each with a different slope, are considered. The final image is obtained by summing the images obtained from each of these plane waves. To obtain an accurate subsurface image, the number of such delayed plane waves is usually less than the number of sources, resulting in computational speedup (Etgen 2006).

In case of delayed shot migration, the effective model is computed in the same manner as described in Algorithm (1) but for a plane-wave source initial condition. Thus, a new effective model is needed for each plane-wave slope. Since the source and receiver wavefields share the same recording surface, the effective model for each plane-wave slope applies to both sources and receivers.

\section{CONCLUSIONS}

Computing effective elliptic models by fitting kinematic features corresponding to the original TTI medium allows us to use the much cheaper elliptic wave extrapolation operators. Using solution of the TTI eikonal equation as a direct source of information for the effective models ensures that we match the phase information of the first arriving wave. For low to moderately complex media, kinematics of later arrivals are also matched with reasonable accuracy. As seen, the amplitude fit is much better for the effective TEA model; however, its cost is higher than solving the VEA model. Thus, the formulations presented here allow us better cost versus accuracy options in estimation of wavefields for anisotropic medium, particularly when it exhibits tilt. An added advantage is that the obtained wavefield solution is free from shear-wave artefacts without the need of any noise suppression filters. Despite using highfrequency asymptotic for matching wavefields, the resulting effective models include most of the critical wavefield components, including frequency dependency and caustics, if present.

\section{ACKNOWLEDGEMENTS}

The authors would like to thank King Abdullah University of Science and Technology for financial support. They are extremely grateful to Ian Jones, Josep de la Puente, and two anonymous reviewers for their valuable comments that greatly helped in improving the text.

\section{REFERENCES}

Alkhalifah T., Ma X., Waheed U. and Zuberi M. 2013. Efficient anisotropic wavefield extrapolation using effective isotropic models. 75th EAGE Conference \& Exhibition incorporating SPE EUROPEC 2013, 10.3997/2214-4609.20130359.
Alkhalifah T. 2000. An acoustic wave equation for anisotropic media. Geophysics 65(4), 1239-1250.

Baysal E., Kosloff D.D. and Sherwood J.W. 1983. Reverse time migration. Geophysics 48(11), 1514-1524.

Crampin S. 1984. An introduction to wave propagation in anisotropic media. Geophysical Journal International 76(1), 17-28.

Etgen J.T. 2006. How many angles do we really need for delayed-shot migration. 68th EAGE Conference \& Exhibition.

Fletcher R.P., Du X. and Fowler P.J. 2009. Reverse time migration in tilted transversely isotropic (TTI) media. Geophysics 74(6), WCA179-WCA187.

Guan H., Dussaud E., Denel B., Williamson P., et al. 2011. Techniques for an efficient implementation of RTM in TTI media. 2011 SEG annual meeting, Society of Exploration Geophysicists.

Huang T., Xu S., Wang J., Ionescu G. and Richardson M. 2008. The benefit of TTI tomography for dual azimuth data in Gulf of Mexico. In: SEG Technical Program Expanded Abstracts 2008. Society of Exploration Geophysicists, 222-226.

Ibanez-Jacome W., Alkhalifah T. and Waheed U. 2014. Effective orthorhombic anisotropic models for wavefield extrapolation. Geophysical Journal International 198(3), 1653-1661.

McMechan G. 1983. Migration by extrapolation of time-dependent boundary values. Geophysical Prospecting 31(3), 413-420.

Shah H. 2007. The 2007 BP anisotropic velocity-analysis benchmark. 70th EAGE annual meeting, workshop.

Stovas A. and Alkhalifah T. 2012. A new traveltime approximation for TI media. Geophysics 77(4), C37-C42.

Tsvankin I. 1997. Moveout analysis for transversely isotropic media with a tilted symmetry axis. Geophysical Prospecting 45(3), 479512.

Vigh D. and Starr E.W. 2008. 3D prestack plane-wave, full-waveform inversion. Geophysics 73(5), VE135-VE144.

Waheed U. and Alkhalifah T. 2014. Effective elliptic models for efficient wavefield extrapolation in anisotropic media. 76th EAGE Conference \& Exhibition, 10.3997/2214-4609.20140813.

Waheed U., Yarman C. and Flagg G. 2014. An iterative fast sweeping based eikonal solver for tilted orthorhombic media. In: SEG Technical Program Expanded Abstracts 2014. Society of Exploration Geophysicists, 10.1190/segam2014-0846.1.

Waheed U., Alkhalifah T. and Stovas A. 2013. Diffraction traveltime approximation for TI media with an inhomogeneous background. Geophysics 78(5), WC103-WC111.

Wang H., Waheed U. and Alkhalifah T. 2014. Effective anisotropy through traveltime and amplitude matching. In: SEG Technical Program Expanded Abstracts 2014. Society of Exploration Geophysicists, doi: 10.1190/segam2014-1183.1.

$\mathrm{Xu}$ S. and Zhou H. 2014. Efficient and accurate algorithm for quasi-P wave propagation. 76th EAGE Conference and Exhibition 2014.

Zhang J.H., Zhang G., Zhang Y., et al. 2009. Removing S-wave noise in TTI reverse time migration. 2009 SEG annual meeting, Society of Exploration Geophysicists.

Zhang Y., Sun J., Notfors C., Gray S.H., Chernis L. and Young J. 2005. Delayed-shot 3D depth migration. Geophysics 70(5), E21E28.

Zhou H., Pham D., Gray S., Wang B., et al. 2004. Tomographic velocity analysis in strongly anisotropic TTI media. 2004 SEG annual meeting, Society of Exploration Geophysicists. 


\section{APPENDIX}

\section{EFFECTIVE VELOCITIES FOR WAVE EXTRAPOLATION IN 3D TTI MEDIA}

In order to derive expressions for effective ellipsoidal media to approximate wavefield solution in a 3D TTI medium, first consider the 3D TTI eikonal equation (Waheed et al. 2013):

$$
\begin{aligned}
& v_{n m o}^{2}(1+2 \eta)\left(\left(\cos \theta \cos \phi \frac{\partial \tau}{\partial x}+\cos \theta \sin \phi \frac{\partial \tau}{\partial y}+\sin \theta \frac{\partial \tau}{\partial z}\right)^{2}+\left(-\sin \phi \frac{\partial \tau}{\partial x}+\cos \phi \frac{\partial \tau}{\partial y}\right)^{2}\right) \\
& +v_{0}^{2}\left(-\sin \theta \cos \phi \frac{\partial \tau}{\partial x}-\sin \theta \sin \phi \frac{\partial \tau}{\partial y}+\cos \theta \frac{\partial \tau}{\partial z}\right)^{2}\left(1-2 \eta v_{n m o}^{2}\left(\left(\cos \theta \cos \phi \frac{\partial \tau}{\partial x}+\cos \theta \sin \phi \frac{\partial \tau}{\partial y}+\sin \theta \frac{\partial \tau}{\partial z}\right)^{2}\right.\right. \\
& \left.\left.+\left(-\sin \phi \frac{\partial \tau}{\partial x}+\cos \phi \frac{\partial \tau}{\partial y}\right)^{2}\right)\right)=1
\end{aligned}
$$

where $\tau(x, y, z)$ is the travel time measured from the source to a point $(x, y, z)$, and $\phi$ is the azimuthal angle. Rewriting the above equation after some algebraic manipulations yields:

$$
\begin{aligned}
& \frac{v_{n m o}^{2}(1+2 \eta)\left(\cos ^{2} \theta \cos ^{2} \phi+\sin ^{2} \phi\right)+v_{0}^{2} \sin ^{2} \theta \cos ^{2} \phi}{c(\tau)}\left(\frac{\partial \tau}{\partial x}\right)^{2}+\frac{v_{n m o}^{2}(1+2 \eta)\left(\cos ^{2} \theta \sin ^{2} \phi+\cos ^{2} \phi\right)+v_{0}^{2} \sin ^{2} \theta \sin ^{2} \phi}{c(\tau)}\left(\frac{\partial \tau}{\partial y}\right)^{2} \\
& +\frac{v_{n m o}^{2}(1+2 \eta) \sin ^{2} \theta+v_{0}^{2} \cos ^{2} \theta}{c(\tau)}\left(\frac{\partial \tau}{\partial z}\right)^{2}=1,
\end{aligned}
$$

where

$$
\begin{aligned}
c(\tau)= & 1+2 \eta v_{n m o}^{2} v_{0}^{2}\left(\left(\cos \theta \cos \phi \frac{\partial \tau}{\partial x}+\cos \theta \sin \phi \frac{\partial \tau}{\partial y}+\sin \theta \frac{\partial \tau}{\partial z}\right)^{2}+\left(-\sin \phi \frac{\partial \tau}{\partial x}+\cos \phi \frac{\partial \tau}{\partial y}\right)^{2}\right) \\
& \times\left(-\sin \theta \cos \phi \frac{\partial \tau}{\partial x}-\sin \theta \sin \phi \frac{\partial \tau}{\partial y}+\cos \theta \frac{\partial \tau}{\partial z}\right)^{2} \\
& -v_{n m o}^{2}(1+2 \eta)\left(\sin 2 \phi\left(\cos ^{2} \theta-1\right) \frac{\partial \tau}{\partial x} \frac{\partial \tau}{\partial y}+\sin 2 \theta \sin \phi \frac{\partial \tau}{\partial y} \frac{\partial \tau}{\partial z}+\sin 2 \theta \cos \phi \frac{\partial \tau}{\partial x} \frac{\partial \tau}{\partial z}\right) \\
& -v_{0}^{2}\left(\sin 2 \phi \sin ^{2} \theta \frac{\partial \tau}{\partial x} \frac{\partial \tau}{\partial y}-\sin 2 \theta \sin \phi \frac{\partial \tau}{\partial y} \frac{\partial \tau}{\partial z}-\sin 2 \theta \cos \phi \frac{\partial \tau}{\partial x} \frac{\partial \tau}{\partial z}\right) .
\end{aligned}
$$

The eikonal equation for ellipsoidally anisotropic eikonal equation is given as:

$v_{n m o 1}^{2}\left(\frac{\partial \tau}{\partial x}\right)^{2}+v_{n m o 2}^{2}\left(\frac{\partial \tau}{\partial y}\right)^{2}+v_{0}^{2}\left(\frac{\partial \tau}{\partial z}\right)^{2}=1$

where $v_{0}$ is the vertical velocity, whereas $v_{n \text { mo } 1}$ and $v_{n \text { mо } 2}$ are the NMO velocities in the $[x, z]$ and $[y, z]$ planes, respectively. Comparing equations (A2) and (A4), we obtain effective velocities for ellipsoidally anisotropic media:

$$
\begin{aligned}
v_{n m o 1, e f f}(x, y, z, s) & =\sqrt{\frac{v_{n m o}^{2}(1+2 \eta)\left(\cos ^{2} \theta \cos ^{2} \phi+\sin ^{2} \phi\right)+v_{0}^{2} \sin ^{2} \theta \cos ^{2} \phi}{c(\tau)}}, \\
v_{n m o 2, e f f}(x, y, z, s) & =\sqrt{\frac{v_{n m o}^{2}(1+2 \eta)\left(\cos ^{2} \theta \sin ^{2} \phi+\cos ^{2} \phi\right)+v_{0}^{2} \sin ^{2} \theta \sin ^{2} \phi}{c(\tau)}}, \\
v_{0, e f f}(x, y, z, s) & =\sqrt{\frac{v_{n m o}^{2}(1+2 \eta) \sin ^{2} \theta+v_{0}^{2} \cos ^{2} \theta}{c(\tau)}},
\end{aligned}
$$

where $v_{\text {nmo } 1, \text { eff }}$ and $v_{\text {nmo2,eff }}$ are effective NMO velocities in the $[x, z]$ and $[y, z]$ planes, respectively, whereas $v_{0, e f f}$ is the effective vertical velocity. 
In a similar manner, for the 3D case, we obtain effective velocities for tilted ellipsoidally anisotropic media to be:

$$
\begin{aligned}
\widetilde{v}_{n m o 1, e f f}(x, y, z, s) & =\widetilde{v}_{n m o 2, e f f}(x, y, z, s)=v_{n m o} \sqrt{\frac{1+2 \eta}{\widetilde{c}(\tau)}}, \\
\widetilde{v}_{0, e f f}(x, y, z, s) & =\frac{v_{0}}{\sqrt{\widetilde{c}(\tau)}},
\end{aligned}
$$

where

$$
\begin{aligned}
\widetilde{c}(\tau)= & 1+2 \eta v_{n m o}^{2} v_{0}^{2}\left(\left(\cos \theta \cos \phi \frac{\partial \tau}{\partial x}+\cos \theta \sin \phi \frac{\partial \tau}{\partial y}+\sin \theta \frac{\partial \tau}{\partial z}\right)^{2}+\left(-\sin \phi \frac{\partial \tau}{\partial x}+\cos \phi \frac{\partial \tau}{\partial y}\right)^{2}\right) \\
& \times\left(-\sin \theta \cos \phi \frac{\partial \tau}{\partial x}-\sin \theta \sin \phi \frac{\partial \tau}{\partial y}+\cos \theta \frac{\partial \tau}{\partial z}\right)^{2} .
\end{aligned}
$$

These velocities can be used in the corresponding wave equation to obtain approximate wavefield solution for a 3D TTI medium. 